\title{
ASI Regulates Satiety Quiescence in C. elegans
}

\author{
Thomas Gallagher, ${ }^{1}$ Jeongho Kim, ${ }^{2}$ Marieke Oldenbroek, ${ }^{3}$ Rex Kerr, ${ }^{4}$ and Young-Jai You ${ }^{1}$ \\ ${ }^{1}$ Department of Biochemistry and Molecular Biology, Virginia Commonwealth University, Richmond, Virginia 23298, ${ }^{2}$ Department of Biological Science, \\ Inha University, Inchon, South Korea, 402-751, 3Department of Molecular Biology, University of Texas Southwestern Medical Center at Dallas, Dallas, \\ Texas 75390, and ${ }^{4}$ Howard Hughes Medical Institute, Janelia Farm Research Campus, Ashburn, Virginia 20147
}

In Caenorhabditis elegans, satiety quiescence mimics behavioral aspects of satiety and postprandial sleep in mammals. On the basis of calciumimaging, genetics, and behavioral studies, here we report that a pair of amphid neurons, ASI, is activated by nutrition and regulates worms' behavioral states specifically promoting satiety quiescence; ASI inhibits the switch from quiescence to dwelling (a browsing state) and accelerates the switch from dwelling to quiescence. The canonical TGF $\beta$ pathway, whose ligand is released from ASI, regulates satiety quiescence. The mutants of a ligand, a receptor and SMADs in the TGF $\beta$ pathway all eat more and show less quiescence than wild-type. The TGF $\beta$ receptor in downstream neurons RIM and RIC is sufficient for worms to exhibit satiety quiescence, suggesting neuronal connection from ASI to RIM and RIC is essential for feeding regulation through the TGF $\beta$ pathway. ASI also regulates satiety quiescence partly through cGMP signaling; restoring cGMP signaling in ASI rescues the satiety quiescence defect of cGMP signaling mutants. From these results, we propose that TGF $\beta$ and cGMP pathways in ASI connect nutritional status to promotion of satiety quiescence, a sleep-like behavioral state.

\section{Introduction}

Appetite control is a complex yet evolutionarily conserved behavior; animals seek food when they are hungry and stop eating and roaming when they are full. Lesions in specific areas of the hypothalamus cause misregulation of appetite (Hetherington and Ranson, 1940; Elmquist et al., 1999), showing that neuronal regulation is critical to control appetite. To understand neuronal regulation of appetite through multiple approaches of genetics, molecular and cellular biology, we use Caenorhabditis elegans, a powerful genetic model with a simple nervous system. Plus we have discovered that $C$. elegans exhibits a behavioral sequence that mimics that of satiety in other animals; after fasting followed by full feeding, animals stop eating and often fall asleep (Antin et al., 1975). This conservation shows that $C$. elegans can be a good model to study appetite control and its neuronal and molecular mechanisms.

Worms' locomotive activity often correlates with their nutritional status (Shtonda and Avery, 2006; Ben Arous et al., 2009). So far, three locomotive states are defined: roaming, dwelling, and quiescence (Fujiwara et al., 2002; You et al., 2008). Roaming correlates with food-seeking and dwelling correlates with browsing. Quiescence is an inactive state where worms do not move and do not eat (You et al., 2008). Several methods to monitor worms'

\footnotetext{
Received Sept. 20, 2012; revised March 25, 2013; accepted April 30, 2013.

Author contributions: T.G., J.K., R.K., and Y.-J.Y. designed research; T.G., J.K., M.O., and Y.-J.Y. performed research; T.G., J.K., R.K., and Y.-J.Y. analyzed data; T.G., R.K., and Y.-J.Y. wrote the paper.

This work was supported by American Heart Association Grant 09SDG2150070 (Y.-J.Y.), NIH Grant 5R01DK083593 (T.G.), Howard Hughes Medical Institute's Janelia Farm Research Campus (R.K.) and Inha University (J.K.). We thank Drs. Ashrafi, Alkema, Beverly, and Sengupta and the Caenorhabditis Genetics Center for strains, Dr. Zimmer for microfluidic devices and Drs. Murakami and Ohshima for the daf-11 DNA construct. We thank Drs. Avery, Bettinger, Davies, and Amir for technical support and invaluable discussion.

The authors declare no competing financial interests.

Correspondence should be addressed to Dr Young-Jai You, Department of Biochemistry and Molecular Biology, Virginia Commonwealth University, 1220 East Broad Street, MMRB Building, Room 2046, Richmond, VA 232980614. E-mail: yjyou@vcu.edu.

DOI:10.1523/JNEUROSCI.4493-12.2013

Copyright $\odot 2013$ the authors $\quad 0270-6474 / 13 / 339716-09 \$ 15.00 / 0$
}

behavior for a prolonged period consistently revealed dwelling and roaming states that correlate with worms' nutritional states. However, there has been no automated method available to measure satiety quiescence because quiescent worms are easily disturbed (Avery, 1993; You et al., 2008). Recently, we developed an automated method of behavioral tracking and hidden Markov model (HMM) analysis to monitor satiety quiescence for prolonged periods of time and then analyze the data to identify the animal's behavioral states incorporating the history. It allowed us to answer questions, such as what the worm's behavioral (and corresponding nutritional) state is at a given time, how long the animal spends in that particular state, whether the animal switches states, and if so, how often (Gallagher et al., 2013).

Here we report the role of ASI in satiety quiescence using the newly developed monitoring system and analysis as well as conventional genetics and calcium imaging. From the new analysis, we found that ASI promotes satiety quiescence by specifically inhibiting the switch from quiescence to dwelling and accelerating the switch from dwelling to quiescence. Using calcium imaging we show that ASI does so by being activated by nutrition, presumably indicating favorable conditions. It is known that ASI releases TGF $\beta$ when the environment is favorable. Indeed, TGF $\beta$ expression is highest when worms show the most consistent quiescence. In addition, all the mutants in the TGF $\beta /$ SMAD pathway are defective in satiety quiescence, and an unbiased genetic screen isolated a TGF $\beta$ receptor mutant deficient in satiety quiescence. ASI also regulates satiety quiescence partly through cGMP signaling; restoring cGMP signaling in ASI rescues the satiety quiescence defect of cGMP signaling mutants. From these results, we propose that TGF $\beta$ and cGMP pathways in ASI connect nutritional status to promotion of satiety quiescence, a sleep-like behavioral state.

\section{Materials and Methods}

General methods and strains. Worms were cultured and handled as described previously (Sulston and Hodgkin, 1988) with the following modifications: 
worms were routinely grown on NGMSR plates (Avery, 1993). All worms were maintained at $20^{\circ} \mathrm{C}$ on Escherichia coli strain HB101 unless indicated otherwise. The wild-type strain was C. elegans variant Bristol, strain N2. Mutant strains used were FK234 egl-4(ks62) IV, DA521 egl-4(ad450sd) IV, CB1372 daf-7(e1372ts) III, CB1393 daf-8(e1393ts) I, DR77 daf-14 (m77) IV, DR40 daf-1 (m40ts) IV, KQ380 daf-1 (m40ts) IV;ftEx205[ptdc-1::daf-1-gfpodr-1::dsRed], DA2316 daf-1 (ad2316) IV, DA2318 daf-1 (ad2316) IV; ftEx205[ptdc-1::daf-1-gfp odr-1::dsRed], DA2228 adEx2228[gpa-4p::egl-4CA rol-6p::GFP], DA2225 adEx2225[tax-4p::egl-4CA rol-6p::GFP], DA2233 egl-4(ks62) IV; adEx2233[gpa-4p::egl-4 rol-6::GFP], DA2145 egl-4(ks62) IV; adEx2145[tax-4::egl-4 rol-6::GFP], DA2313 tdc-1(ok914) II; daf-7(e1372ts) III, DR47 daf-11(m47) V, DA2221 daf-11(sa195ts) V; adEx2221 [gpa-4p::daf-11 rol-6p::GFP], DA2258 daf-7(e1372ts) III; adEx2258 [tax-4p::egl-4gf rol-6p::GFP], DA2258 daf-7(e1372ts) III; adEx2258[tax-4p::: egl-4gf rol-6p::GFP], DA2221 daf-11(sa195ts) V; adEx2221 [gpa-4p::daf-11 rol-6p::GFP], DA2230 adEx2230[gpa-4p::egl-4(gf) rol-6p::GFP] KQ280 daf1(m40ts) IV; ftEx98[pdaf-1::daf-1-gfp odr-1::dsRED], KQ324 daf-1(m40ts) IV; ftEx175[pB0280.7::daf-1-gfp odr-1::dsRED], KQ275 daf-1(m40ts) IV; ftEx93[pglr-1::daf-1-gfp odr-1::dsRED], KQ251 daf-1(m40ts) IV; ftEx69[pegl-3:: daf-1-gfp odr-1::dsRED], KQ265 daf-1(m40ts) IV; ftEx83[posm-6::daf-1-gfp odr-1::dsRED], KQ315 daf-1 (m40ts) IV; ftEx166[pflp-1::daf-1-gfpodr-1::dsRED], KQ380 daf-1 (m40ts) IV; ftEx205[ptdc-1::daf-1-gfp odr-1::dsRED], KQ332 daf1(m40ts) IV; ftEx183[pglr-7::daf-1-gfp odr-1::dsRED], PY7505 (Beverly et al., 2011), DA2227 adEx2227 [gpa-4p::GFP], PY6554 (Beverly et al., 2011), YJ87 egl4(ks62) IV; uyEx87[gpa-4p::GCaMP2.2b unc-122::dsRed], YJ88 egl-4(ad450sd) IV; uyEx88[gpa-4p::GCaMP2.2b unc-122::dsRed], and DA2287 adEx2287 [daf-7p:: daf-7::mCherry daf-7p::GFP].

Molecular biology. Generation of a constitutively active cGMPdependent protein kinase (EGL-4CA): primers YJ323f (GCC AAA AAG ATT GCC GTT GAT GCA GAG CCG ACG) and YJ324r (CGT CGG CTC TGC ATC AAC GGC AAT CTT TTT GGC) were used to introduce an amino acid change from Ser to Asp so as to generate constitutively active C. elegans PKG, EGL-4. The mutated sequences are shown in bold. The corresponding sequence in plasmids containing egl-4 cDNA with either a tax-4 or a $g p a-4$ promoter was replaced with the mutant PCR fragment (You et al., 2008).

Mutagenesis and isolation of mutants. Approximately $10^{4} \mathrm{P}_{0} \mathrm{~L} 4 \mathrm{~s}$ were mutagenized with EMS (ethylmethanesulfonate) for $4 \mathrm{~h}$ and $\sim 6000 \mathrm{~F}_{2} \mathrm{~s}$ were screened for defects in satiety quiescence (Sulston and Hodgkin, 1988). As a primary screen, synchronized well fed 1-d-old adults were fasted in $3 \mathrm{ml}$ of M9 liquid for $12 \mathrm{~h}$ and refed for 3-6 h, during which time the phenotype was scored individually. The defect in quiescence was confirmed by repeating the assay as described previously (You et al., 2008); 10 worms were tested for each mutant and the assay was repeated three times independently.

Satiety quiescence and food intake assay. Satiety quiescence and food intake were measured as described previously (You et al., 2008; Raizen et al., 2012). Briefly, for the "fasted and refed" test, worms were fasted for $12 \mathrm{~h}$ and refed for 3 or $6 \mathrm{~h}$ to examine satiety quiescence. Once worms were found to be quiescent, the duration was measured for 10 worms then averaged. For the "nonfasted " test, 10 worms for each test group were picked singly as L4s into HB101 seeded $35 \mathrm{~mm}$ NGMSR plates and left for $18 \mathrm{~h}$ without disruption. Then the quiescence durations were measured. All experiments were repeated at least three times and one representative graph is shown. To measure food intake, mCherry-expressing E. coli strain $\mathrm{HB} 101$ was inoculated in $\mathrm{LB}$ and grown overnight at $37^{\circ} \mathrm{C}$, and then seeded on $35 \mathrm{~mm}$ NGMSR plates and incubated overnight at $37^{\circ} \mathrm{C}$. Plates were stored at room temperature for at least one night. Worms were fasted and refed as described above (You et al., 2008). After $3 \mathrm{~h}$ of refeeding, worms were treated with $100 \mu \mathrm{l}$ of $1 \mathrm{M}$ sodium azide for their feeding status to be fixed. Worms were observed using a Zeiss Axio A2 Imager with a $10 \times$ objective lens. Images were acquired using Zeiss Axiovision software and fluorescence was quantified using ImageJ.

Quantifying daf-7 expression. Worms (DA2287) expressing daf-7 tagged with mCherry under the daf-7 promoter were prepared identically to satiety quiescence assays described above. Worms were collected by treating with $1 \mathrm{~m}$ sodium azide $\left(\mathrm{NaN}_{3}\right)$, transferred to an agar pad, and observed using a Zeiss Axio A2 Imager with a $100 \times$ objective lens. Images were acquired using Zeiss Axiovision software and fluorescence was quantified using ImageJ.
Preparation of bacteria for locomotion assays. Five milliliters of LB was inoculated with a single colony of E. coli strain HB101 expressing mCherry and incubated shaking overnight at $37^{\circ} \mathrm{C}$. The culture was removed from the incubator and allowed to sit at room temperature overnight. The sample was centrifuged at $4000 \mathrm{rpm}$ for $3 \mathrm{~min}$. After decanting the supernatant, the pellet was resuspended in the small residual amount of broth and transferred to a microcentrifuge tube. Forty microliters of this suspension was twice serially diluted 1:1 with M9 (for a final $4 \times$ dilution). Five microliters of this suspension was pipetted onto a $35 \mathrm{~mm}$ NGMSR plate and allowed to dry completely.

Aztreonam was used to prepare low-quality food (Ben Arous et al., 2009). Aztreonam prevents bacterial cell division, so that the bacteria turn into long strands, which are difficult for the worm to swallow. Aztreonam-treated bacteria were prepared as above with one additional step. After shaking overnight at $37^{\circ} \mathrm{C}, 1 \mathrm{ml}$ of the culture was added to 4 $\mathrm{ml}$ of fresh LB and aztreonam (Sigma-Aldrich) was added to a final concentration of $5 \mu \mathrm{g} / \mathrm{ml}$. This was incubated overnight shaking at $37^{\circ} \mathrm{C}$, and then allowed to sit at room temperature overnight.

Locomotion monitoring assays. L4 worms were picked to a HB101 seeded NGMSR plate and given $8 \mathrm{~h}$ to develop to young adults. Young adult worms (adults containing no eggs) were picked to individual $60 \mathrm{~mm}$ NGMSR plates without food and starved for $12-14 \mathrm{~h}$. A single starved worm was then transferred to a $\sim 6$-mm-diameter spot of bacteria made by placing $5 \mu \mathrm{l}$ of bacterial culture on a plate (described above), focused under the camera, and allowed to refeed for $3 \mathrm{~h}$. The microscope light was then turned on and video capture was started at 1 frame/s for $1 \mathrm{~h}$.

For nonfasted assays, worms were prepared as described above except that young adults were transferred to a HB101 seeded NGMSR plate for 12-14 h. After transfer worms were given $30 \mathrm{~min}$ on the assay plate to recover, followed by taking a $30 \mathrm{~min}$ video at $1 \mathrm{frame} / \mathrm{s}$.

Worms were recorded using a Leica MZ6 microscope at $2.5 \times$ magnification with a $1.0 \times$ lens and a Retiga-4000R camera and Image Pro Plus 6.2. Locomotion videos were analyzed by Image Pro Plus software. (This analysis has been repeated using a custom written MatLab program). A low pass filter was applied to each frame of the movie and the light/dark threshold was adjusted to find the outline of the worm. The center of mass was calculated at each time, reducing each recording to a series of $(t$, $x, y)$ points, which were the basis for all subsequent analyses.

A certain amount of motion is detected even from a completely stationary worm, as small fluctuations in measured brightness of border pixels cause them to vary above and below threshold. This noise motion places a limit on our ability to detect immobility and therefore quiescence. To quantify it, we recorded a worm immobilized with $30 \mu \mathrm{l} \mathrm{of} 1 \mathrm{M}$ $\mathrm{NaN}_{3}$ before transfer to the assay plate. The mean measured speed of an immobilized worm was $0.32 \mu \mathrm{m} \mathrm{s}^{-1}$, and the speed was $<1 \mu \mathrm{m} \mathrm{s}^{-1}$ $99.7 \%$ of the time. Apparent motion was biased along one direction, as expected, because most border pixels are farther from the center in the anterior/posterior direction than in the dorsal/ventral direction.

ASI ablation by laser killing. DA2227 adEx2227[gpa-4p::GFP] was used to identify ASI and KQ380 daf-1(m40ts) IV; ftEx205[ptdc-1::daf-1-gfp $o d r-1:: d s R E D]$ was used to identify RIM and RIC from GFP. The neurons were ablated in L1s within $4 \mathrm{~h}$ of hatching on food (Bargmann and Avery, 1995). The worms were recovered and the ablation was confirmed the next day by lack of fluorescence in ASI. Mock-ablated control worms were treated identically except that the laser was not fired.

Calcium imaging. All calcium imaging experiments were performed on an Olympus BX51 upright microscope with a long-working-distance $40 \times$ water-immersion objective and a Photometrics Evolve 128 EMCCD camera. Analysis of the imaging data were performed with a custom Java-based program as in Suzuki et al. (2003) with one region of interest placed on ASI and a second placed nearby to measure background.

Young adult worms were picked and placed in a microfluidic device that restrains the worm with the tip of the head (where the ASI sensory neurons are located) in a stream that can be rapidly switched (Chronis et al., 2007) ("the olfactory chip"). Images were recorded at 100 frames/s for $60 \mathrm{~s}$. Each worm was recorded for a $15 \mathrm{~s}$ baseline, followed by exposure to stimulus for $15 \mathrm{~s}, 15 \mathrm{~s}$ no stimulus, and a second $15 \mathrm{~s}$ exposure to stimulus.

Body size measurement. L4 stage worms were picked to HB101 seeded plates under fluorescence to sort transgenic and nontransgenic worms. 
The worms were grown at $20^{\circ} \mathrm{C}$ for $24 \mathrm{~h}$ (day one adults) or $48 \mathrm{~h}$ (day two adults). To measure body size, worms were transferred to a plate with no bacteria and imaged using a Zeiss Discovery V8 microscope and a Point Gray RoHS 1.4MP B and W Grasshopper 1394b Camera at 7.5 frames/s. The area of each worm was calculated using a custom written MatLab program. The area of the worm in seven consecutive frames was calculated and the result averaged.

Statistics. All bar graphs denote mean \pm SEM. Statistical tests were done using MatLab, Mathematica, and $\mathrm{R}$ programming tools.

\section{Results}

Animals switch states depending on nutritional status

To understand the neuronal mechanisms of satiety quiescence, we developed an automated procedure to monitor a single worm's behavior over long time periods (Gallagher et al., 2013) (see Materials and Methods). We previously showed that satiety quiescence is suppressed by low-quality food, enhanced by high-quality food, enhanced by fasting followed by refeeding, and suppressed by egl-4 loss-of-function mutations as measured by quiescence durations after 3 and $6 \mathrm{~h}$ of refeeding following a $12 \mathrm{~h}$ fast (You et al., 2008). When we monitored an individual worm's locomotive activity for an hour after $3 \mathrm{~h}$ of refeeding using our newly developed system, the same treatments caused the expected results; fed with low-quality food, worms move more than the worms fed with high-quality food as shown their average speed over an hour during refeeding. (Fig. $1 A-F$ ). In addition, worms were completely inactive for significant durations, confirming that satiety quiescence occurred under our recording conditions. Therefore our automated procedure confirmed that worms change their locomotive activity depending on their nutritional status and that quiescence is a behavioral state that occupies a significant portion of the worms' life under certain conditions. Most importantly, our new method of monitoring worms' locomotive activity for an hour during refeeding reveals previously unknown phenomena; there is a clear alternation of periods of prolonged inactivity (indicated as gray bars) and continuous activity (indicated as black bars). This suggests a potential cyclicity of behavioral states (Fig. 1G).

\section{TGF $\beta$ in ASI promotes the switch from dwelling} to quiescence

To capture the information available in the time course of behavior so as to understand how animals' behavior changes over the time, we used an HMM (Gallagher et al., 2013). To directly compare the effects of genotype and experimental con-

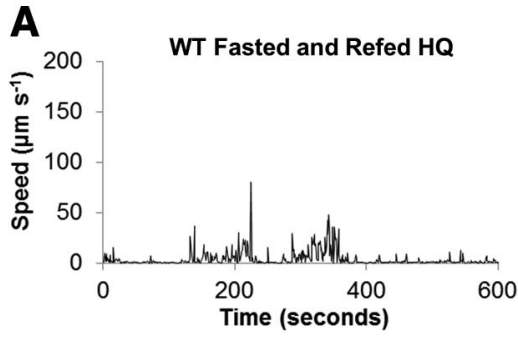

B

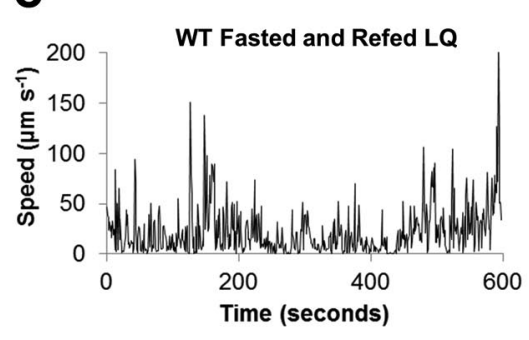

D
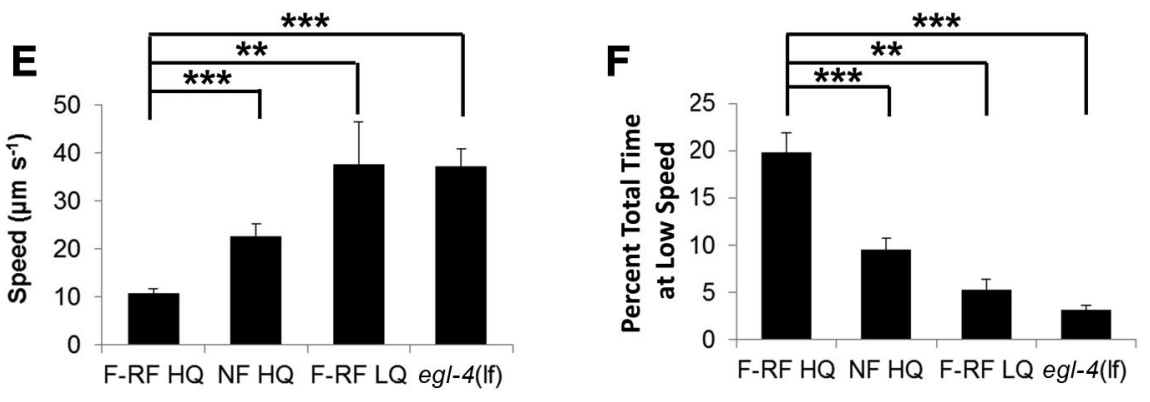

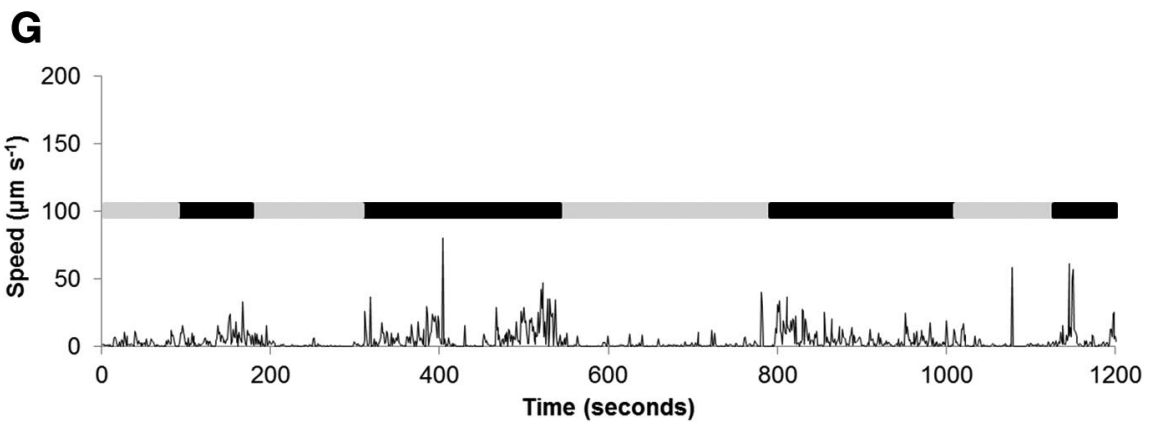

Figure 1. Locomotive activity represents nutritional status of animals. $A$, A representative speed time course of a fasted and refed wild-type worm on high quality food. $B$, A representative speed time course of a nonfasted wild-type worm on high quality food. $C$, A representative speed time course of a fasted and refed wild-type worm on low quality food. $\boldsymbol{D}$, A representative speed time course of a fasted and refed egl- 4 loss of function mutant on high quality food. $\boldsymbol{E}$, The mean speed of the worm in each experiment was calculated and the average taken to give the mean speed of worms for each of the four conditions listed above $(\boldsymbol{A}-\boldsymbol{D}) . \boldsymbol{F}$, The percentage total time that worms spend with the speed of $<1 \mu \mathrm{m} \mathrm{s}^{-1}$ from the conditions 1A-1D. E, F, F-RF HQ: fasted and refed high quality food, NF HQ: nonfasted and fed high quality food. F-RF LQ: fasted and refed low quality food. egl-4(If): an egl-4 loss of function mutant fasted and refed high quality food. Following Kruskal-Wallis ANOVA ( $p<0.001$ for both), ${ }^{*} p<$ $0.05,{ }^{* *} p<0.01,{ }^{* * *} p<0.001$, by Mann-Whitney $U$ test. In each experiment, each worm was monitored for 30 min if nonfasted or $1 \mathrm{~h}$ if fasted and refed. At least 10 worms were used for each condition. G, Locomotive activity for $20 \mathrm{~min}$ during the course of refeeding shows a pattern of alternating periods of active (black bar) and inactive (gray bar). For this simple determination of active and inactive, states were determined subjectively.

ditions on locomotion behavior, we developed standard descriptions of roaming, dwelling, and quiescence states that could be used for analysis of all tracks (Gallagher et al., 2013). From the analysis, we found that under the most favorable conditions for satiety quiescence (after fasting followed by refeeding), wild-type worms spent $59 \pm 3 \%$ of the time quiescent and most or all of the remaining time $(41 \pm 3 \%)$ dwelling (Fig. $2 A)$. Mutations in $d a f-7$ which encodes a worm TGF $\beta$ signifi- 
A

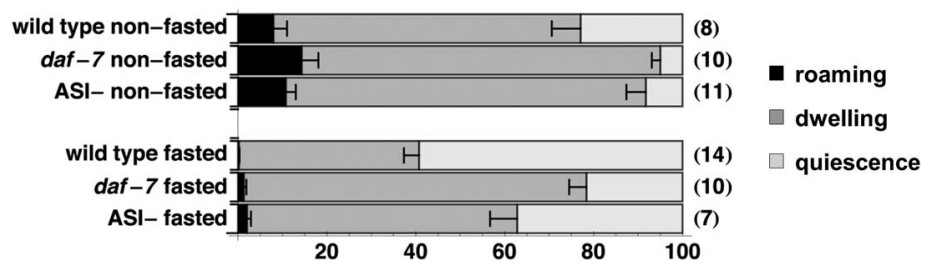

B

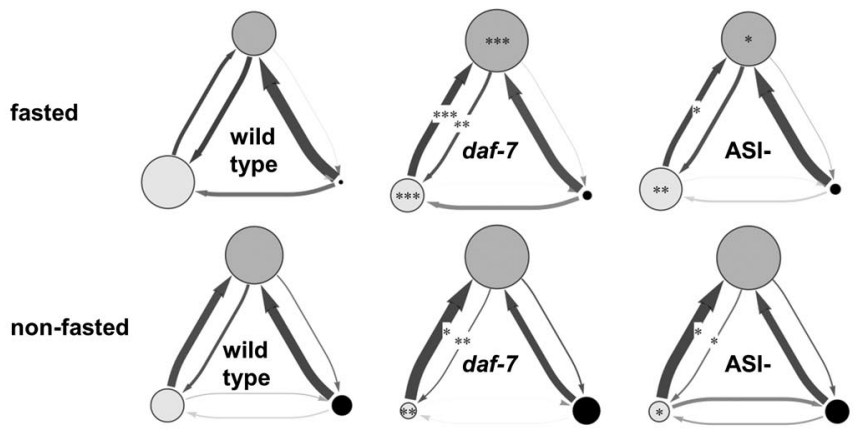

D

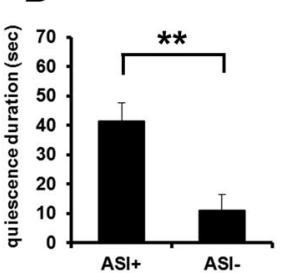

G

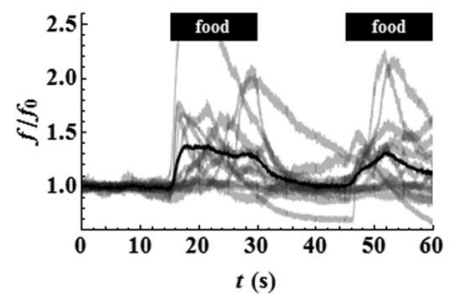

E

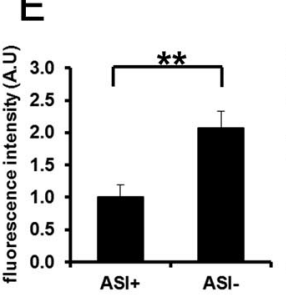

$\mathrm{F}$

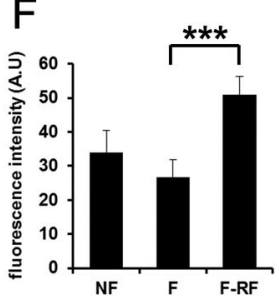

$\mathrm{H}$

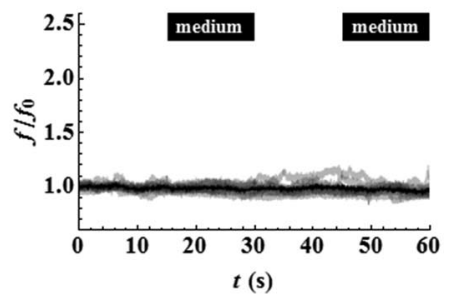

Figure 2. TGF $\beta$ in ASI neurons promote the switch from dwelling to quiescence. $A$, Standard state probabilities ( $x$-axis) of wild-type, daf-7 mutants and worms whose ASI neurons were genetically ablated by the recCaspase method (Chelur and Chalfie, 2007; Beverly et al., 2011) (ASI -). Numbers in the parenthesis are the sample size. Error bars are SEM. B, DAF-7 from ASI regulates transition rates from dwelling to quiescence. Transition rates among states of wild-type, daf-7 mutants, and worms whose ASI neurons were genetically ablated by the recCaspase method (Chelur and Chalfie, 2007; Beverly et al., 2011). Each diagram shows state probabilities and transition rates from one experiment after standard state fits. Each circle represents a state: light gray for quiescence, medium gray for dwelling, and black for roaming. The area of the circle is proportional to the probability of the state under those conditions. Each arrow represents a transition from one state to another. Thicker arrows represent higher transition rates. Darker arrows represent rates measured with high accuracy, paler arrows rates measured with poor accuracy. ${ }^{*} p<0.05$, ${ }^{* *} p<0.01,{ }^{* * *} p<0.001$, by Mann-Whitney $U$ test. C, Duration of satiety quiescence of mock operated worms (ASI + ) and worms whose ASI neurons were killed with a laser (ASI-). Worms were fasted for $12 \mathrm{~h}$ and refed for $6 \mathrm{~h}$ then the quiescence durations were measured. $n=10$ for each group per experiment, repeated three times. ${ }^{*} p<0.05,{ }^{* *} p<0.01,{ }^{* * *} p<0.001$, by Student's $t$ test. $\boldsymbol{D}$, Duration of satiety quiescence of worms whose ASI neurons were genetically ablated by the recCaspase method (Chelur and Chalfie, 2007; Beverly et al., 2011). ASI - worms showed reduced satiety quiescence. $n=10$ for each group per experiment, repeated three times. ${ }^{* *} p<0.01$, by Student's $t$ test. $\boldsymbol{E}$, Worms with ASI genetically ablated ate more than worms with intact ASI when food intake was measured with mCherry-expressing $E$. coli after $3 \mathrm{~h}$ of refeeding followed by $12 \mathrm{~h}$ of starvation. The ASI - worm had more fluorescence in the gut than ASI +, reflecting higher food intake. $n=10$ for each group per experiment, repeated three times. ${ }^{* *} p<0.01$, by Student's $t$ test. $F$, DAF- 7 is upregulated after fasting and refeeding. We measured DAF-7 expression by quantifying fluorescence in the head of worms expressing DAF-7 fused to $\mathrm{mCherry} \mathrm{in} \mathrm{worms} \mathrm{not}$ fasted (NF), fasted for $12 \mathrm{~h}$ (F), or fasted for $12 \mathrm{~h}$ and refed for $3 \mathrm{~h}$ (F-RF). $n=10$ for each group per experiment, repeated four times. The graphs show the average of all experiments. ${ }^{* * *} p<0.001$, Kruskal-Wallis ANOVA $\left(^{* * *} p<0.001\right)$ followed by MannWhitney $U$ test. $\mathbf{G}-H_{,} \mathrm{Ca}^{2+}$ imaging from ASI neurons. One or both ASIs were imaged as either control or an experimental stimulus flowed past the tip of the head, where the ASI sensory endings are located. The stimulus, either E. coli HB101 in minimal medium (G) or minimal medium alone $(\boldsymbol{H})$, was presented from 15 to $30 \mathrm{~s}$ and again from 45 to $60 \mathrm{~s}$. Individual traces, normalized so that the mean for the first $15 \mathrm{~s}$ (before presentation of stimulus) is 1 , are shown in light gray ( is fluorescence, $\mathrm{f}_{0}$ baseline fluorescence; the ratio is $\mathrm{f} / \mathrm{f}_{0 \text { ). }}$. The dark black line is the mean of the normalized traces. $n=14$ for stimulus, $n=10$ for control.

cantly reduce satiety quiescence (You et al., 2008). daf-7 mutants spent only $22 \pm 4 \%$ of the time quiescent and most of the rest $(77 \pm 4 \%)$ dwelling. Interestingly, daf-7 mutants showed enhanced dwelling even when well fed: daf-7 mutants spent $81 \pm 4 \%$ of the time dwelling, compared with $69 \pm 5 \%$ for wild-type (Fig. 2A). This difference was due to a decrease in the time spent quiescent in both conditions; there was no significant change in roaming (Fig. $2 B$ ). daf-7 mutants exhibit enhanced dwelling but reduced quiescence because quiescent daf-7 worms switch to dwelling more rapidly than quiescent wild-type worms, and dwelling daf-7 worms switch to quiescence more slowly than dwelling wildtype worms (Fig. 2B).

DAF-7 is released by ASI neurons in response to conditions that are favorable for growth (Ren et al., 1996). Genetic or laser killing of ASI neurons had effects on satiety quiescence and food intake similar to that of mutating daf-7 (Fig. 2C-E) (see Materials and Methods), suggesting that ASI is the source of DAF-7 release. This implies that ASI promotes the switch from dwelling to quiescence and inhibits the switch from quiescence to dwelling, at least in part by releasing DAF-7. We measured DAF-7 synthesis in ASI in well fed, fasted and fasted-refed worms using DAF-7 protein fused with mCherry. As shown in Figure $2 F$, the protein levels measured by intensity of fluorescence mostly coming out from ASI slightly decreased in fasted worms compared with well fed worms. Importantly, the level of DAF-7 is significantly increased after refeeding compared with either well fed or fasted. We speculate the reason we saw little change in fluorescence in fasted worms compared with well fed is the stability of the mCherry protein; it is not degraded fast enough to show the difference (unpublished data). However, refeeding significantly increases the fluorescence clearly showing that DAF-7 is newly synthesized during refeeding. This, with the previous study (Ren et al., 1996), strongly suggests that DAF-7 level correlates with nutritional state.

Under adverse conditions, C. elegans larvae can enter a developmental diapause known as the dauer larva ( $\mathrm{Hu}, 2007)$. Food (Golden and Riddle, 1984a), ASI (Bargmann and Horvitz, 1991), and DAF-7 (Ren et al., 1996) inhibit dauer formation. These facts together with our results above and previously described effects of daf-7 on behavior (You et al., 2008; Ben Arous et al., 2009) suggested that ASI might respond to the worm's nu- 
tritional state to regulate satiety quiescence. We tested this prediction by $\mathrm{Ca}^{2+}$ imaging.

ASI was activated by bacteria grown in minimal medium (Fig. $2 G$ ) but not by minimal medium alone (Fig. $2 H$ ) or by freshly washed bacteria (data not shown). ASI was also activated by LB (data not shown), which contains a mixture of soluble nutrients. This result suggested that ASI, by responding to nutritional signals but not to bacteria themselves, can sense external nutrients, and possibly by extension internal nutrients, to promote the switch from dwelling to quiescence.

\section{Canonical TGF $\beta$ signaling regulates food intake and} satiety quiescence

To understand the role of canonical TGF $\beta$ signaling in satiety quiescence, we tested satiety quiescence of TGF $\beta$ signaling pathway mutants using the method as previously described (You et al., 2008). Previously, we have shown that the ligand (DAF-7) and the receptors (DAF-1 and DAF-4) of this TGF $\beta$ signaling pathway are necessary for full satiety quiescence (You et al., 2008). Upon binding of ligands, TGF receptor type I and II are activated and phosphorylate their downstream SMADs (Patterson and Padgett, 2000). To test whether the canonical TGF $\beta$ pathway regulates satiety quiescence, we tested satiety quiescence of mutants of daf-8 and daf-14, the SMAD transcription factors downstream of DAF-1 and DAF-7. Both are similarly defective in satiety quiescence to daf-7 mutants (Fig. $3 A, B$ ) (You et al., 2008). To test whether mutants in TGF $\beta$ pathway are also defective in controlling food intake, we measured their food intake of them using mCherry expressing $E$. coli as food. After $12 \mathrm{~h}$ of starvation followed by $3 \mathrm{~h}$ of refeeding, food intake is increased in all TGF $\beta$ pathway mutants compared with wild-type (Fig. $3 C-G$ ), confirming that the canonical TGF $\beta$ pathway regulates satiety quiescence and food intake.

To find genes that regulate satiety quiescence, we performed an unbiased genetic screen for mutants defective in satiety quiescence (see Materials and Methods). Among 11 isolates, one mutant not only shows reduced satiety quiescence compared with wild-type (Fig. $3 \mathrm{H}$ ), but also produces dauers, has dark intestine, and shows a bordering phenotype (data not shown). All these phenotypes are similar to those of known TGF $\beta$ pathway mutants. After complementation tests with known Daf-c mutants of the TGF $\beta$ pathway, we identified it as daf-1. The new daf- 1 allele carries a glycine to glutamate mutation in position 110 (G110E). An existing allele $m 122$ also contains the same mutation in addition to E105A (Gunther et al., 2000), showing that Glycine 110 is critical for DAF-1 function. Together, our results confirm that the canonical TGF $\beta$ pathway regulates satiety quiescence and food intake.

\section{DAF-7 TGF $\beta$ regulates satiety quiescence through RIM and RIC neurons}

To ask which neurons act downstream of ASI to regulate satiety quiescence, we took advantage of the satiety quiescence defect of daf-1 mutants. Greer et al. (2008) found that expressing DAF-1 in RIM and RIC neurons is sufficient to rescue daf-1 phenotypes in dauer formation, pumping, fat storage and egg laying. We tested a series of transgenic lines of daf-1 (Table 1, courtesy of Kaveh Ashrafi) that target expression of DAF-1 to different neurons. DAF-1 in RIM and RIC neurons was sufficient to rescue the daf-1 defect in satiety quiescence (Fig. 4A). The same rescue construct rescued the new daf- 1 allele ad2316 (Fig. 4B).

RIM and possibly RIC are thought to be activated during starvation (Alkema et al., 2005; Suo et al., 2006; Greer et al., 2008; Crocker et al., 2010). We therefore hypothesized that the binding
A
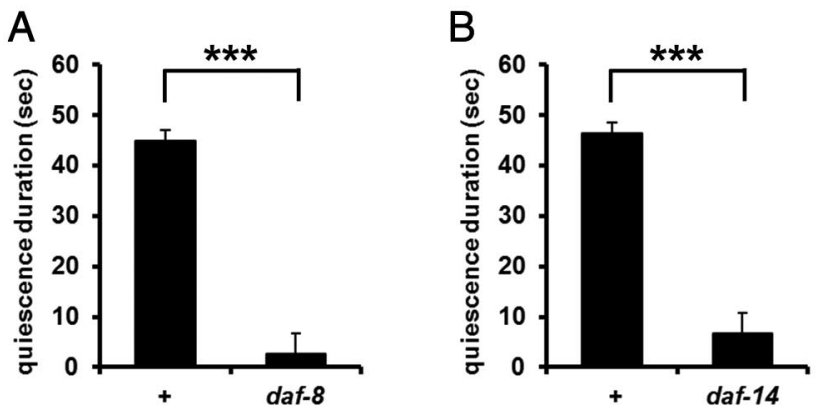

C

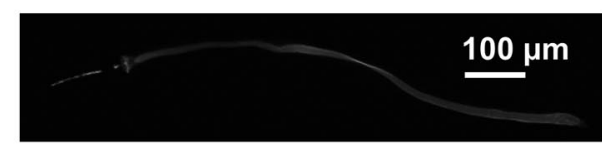

D

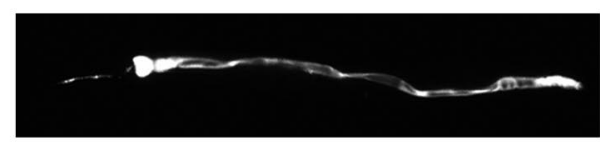

$\mathrm{E}$
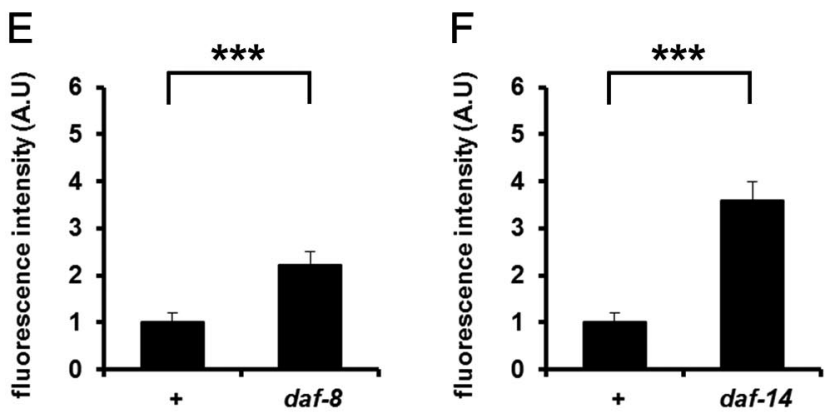

\section{G}

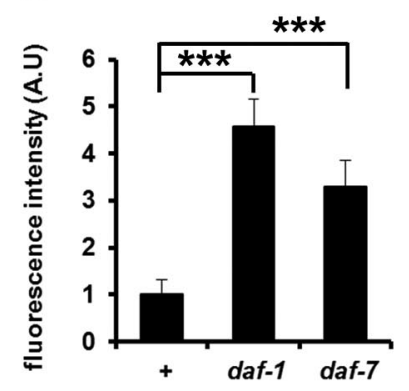

$\mathrm{H}$

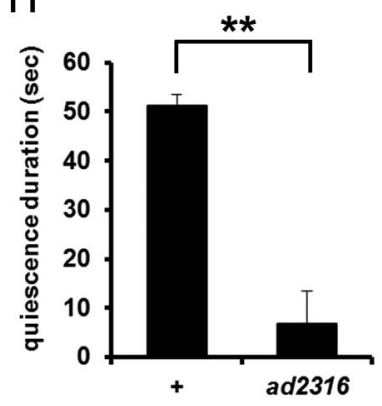

Figure 3. Canonical TGF $\beta$ signaling regulates food intake and satiety quiescence. $\boldsymbol{A}$, daf- 8 mutants had reduced satiety quiescence compared with wild-type $(+) . n=10$ for each group per experiment, repeated three times. ${ }^{* * *} p<0.001$, by Student's $t$ test. $\boldsymbol{B}$, daf-14 mutants had reduced satiety quiescence compared with wild-type $(+) . n=10$ for each group per experiment, repeated three times ${ }^{* * *} p<0.001$, by Student's $t$ test. $C, D$, Representative pictures of a wild-type $(\boldsymbol{C})$ and a daf-7 mutant $(\boldsymbol{D})$ worm after refeeding with $\mathrm{mCherry}$-expressing $E$. coli after $3 \mathrm{~h}$ of refeeding followed by $12 \mathrm{~h}$ of starvation. The daf-7 mutant had more fluorescence in the gut than wild-type, reflecting higher food intake. $\boldsymbol{E}-\boldsymbol{G}$, Canonical TGF $\beta$ pathway mutants ate more than wild-type $(+)$ when food intake was measured by mCherry-expressing $E$. coli. $n=10$ for each group. $\boldsymbol{E}$, daf-8, a smad mutant; $\boldsymbol{F}$, daf-14, a smad mutant; $\boldsymbol{G}$, daf-7, the ligand mutant and daf-1, the type I receptor mutant. Each mutant is shown with a concurrent wild-type control. $n=10$ for each group per experiment, repeated at least three times. $\boldsymbol{E}, \boldsymbol{F}^{* * *} p<0.001$, by MannWhitney $U$ test. $\boldsymbol{G}^{* * *} p<0.001$, Kruskal-Wallis ANOVA $(p<0.001)$ followed by MannWhitney $U$ test. $\boldsymbol{H}$, A new daf- 1 mutant isolated through an unbiased screen for defects in satiety quiescence. $n=10$ for each group per experiment, repeated three times. 
Table 1. Satiety quiescence of daf-1 rescue lines

\begin{tabular}{|c|c|c|c|c|c|c|}
\hline \multirow[b]{2}{*}{ Strains* } & \multirow[b]{2}{*}{ Promoters* } & \multirow[b]{2}{*}{ Target neurons* } & \multicolumn{3}{|c|}{ Satiety quiescence duration (sec) } & \multirow[b]{2}{*}{$p$ value ( $t$ test) } \\
\hline & & & Nontransgenic & Transgenic & Wild-type & \\
\hline KQ280 & daf-1 & many $(>80)$ neurons & $8.2 \pm 5.5$ & $42.2 \pm 15.0$ & $52.2 \pm 3.5$ & 0.07 \\
\hline KQ324 & B0280.7 & 5 pharyngeal gland & $15.2 \pm 4.3$ & $9.9 \pm 6.0$ & $51.8 \pm 4.2$ & 0.47 \\
\hline KQ275 & $g / r-1$ & 24 including RIM & $18.6 \pm 6.7$ & $40.7 \pm 10.3$ & $59.3 \pm 7.3$ & 0.1 \\
\hline KQ251 & egl-3 & many $(>80)$ neurons & $11.1 \pm 5.4$ & $44.5 \pm 10.5$ & $43.4 \pm 2.3$ & 0.07 \\
\hline KQ265 & osm-6 & 56 ciliated neurons & $24.9 \pm 4.9$ & $17 \pm 5.8$ & $65.2 \pm 6.4$ & 0.31 \\
\hline KQ315 & $f l p-1$ & 2 head neurons & $22 \pm 5.2$ & $42.5 \pm 7.7$ & $52.8 \pm 5.4$ & 0.04 \\
\hline KQ380 & $t d c-1$ & RIM, RIC & $16.7 \pm 2.6$ & $68.9 \pm 6.2$ & $53.2 \pm 4.3$ & 0.002 \\
\hline KQ332 & $g / r-7$ & 9 pharyngeal neurons & $24.8 \pm 5.8$ & $20.9 \pm 6.2$ & $60.4 \pm 7.5$ & 0.65 \\
\hline
\end{tabular}

*Greer et al., 2008.

A

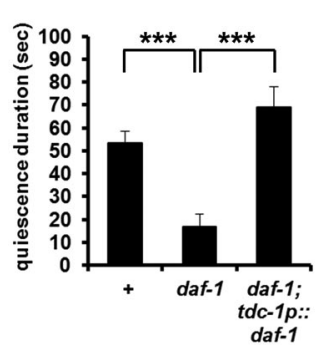

C

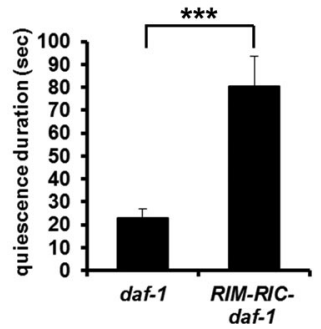

B

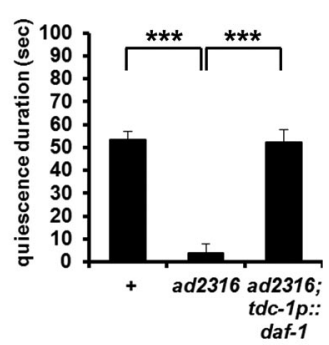

D

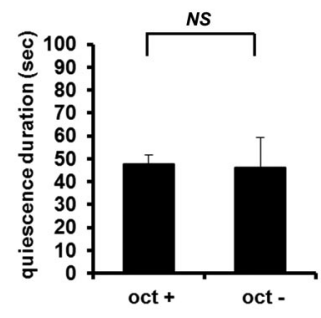

Figure 4. DAF-7 TGF $\beta$ regulates satiety quiescence through RIM and RIC neurons. $\boldsymbol{A}$, daf$1(+)$ expression in RIM and RIC neurons of a daf- 1 mutant rescued its defect in satiety quiescence. $n=10$ for each group per experiment, repeated three times. ${ }^{* * *} p<0.001$, by Student's $t$ test. $\boldsymbol{B}$, daf- 1 expression in RIM and RIC rescued the newly isolated daf- 1 allele, ad2316. $n=$ 10 for each group per experiment, repeated three times. ${ }^{* * *} p<0.001$, by Student's $t$ test. $\boldsymbol{C}$, Laser ablation of RIM and RIC in a daf- 1 mutant restored normal satiety quiescence. $n=10$ for each group per experiment, repeated three times. ${ }^{* * *} p<0.001$, by Student's $t$ test. $\boldsymbol{D}$, Acute treatment with octopamine $(5 \mathrm{~mm})$ does not inhibit satiety quiescence. Octopamine plated on top of the agar before the quiescence test. Worms were treated with octopamine for $6 \mathrm{~h}$ of refeeding period only. $n=10$ for each group per experiment, repeated three times. NS, Not significant by Student's $t$ test.

of DAF-7 to DAF-1 in RIM and RIC inactivates the neurons when nutrients are abundant, and that this inhibition of RIM and RIC is necessary for satiety quiescence. To test this idea, we killed RIM and RIC neurons in daf-1 mutants with a laser. Killing RIM and RIC rescued satiety quiescence (Fig. $4 C$ ).

Octopamine is one of several neurotransmitters and peptide signals released by RIM or RIC (Alkema et al., 2005). Octopamine, sometimes thought of as the invertebrate equivalent of norepinephrine, is known to regulate certain aspects of the behavioral response to starvation in worms and to regulate sleep in flies (Avery, 1993; Suo et al., 2006; Crocker et al., 2010). If RIM and RIC inhibit quiescence by release of octopamine, it might be possible to mimic the daf- 1 defect by adding octopamine. However, acute treatment with octopamine $(5 \mathrm{~mm})$ during the refeeding period did not inhibit satiety quiescence (Fig. 4D). The failure of acute octopamine treatment to suppress quiescence suggests either that different conditions are necessary for octopamine to function as a star-

A

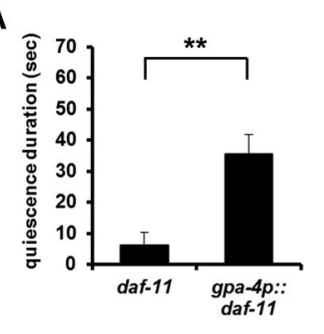

B
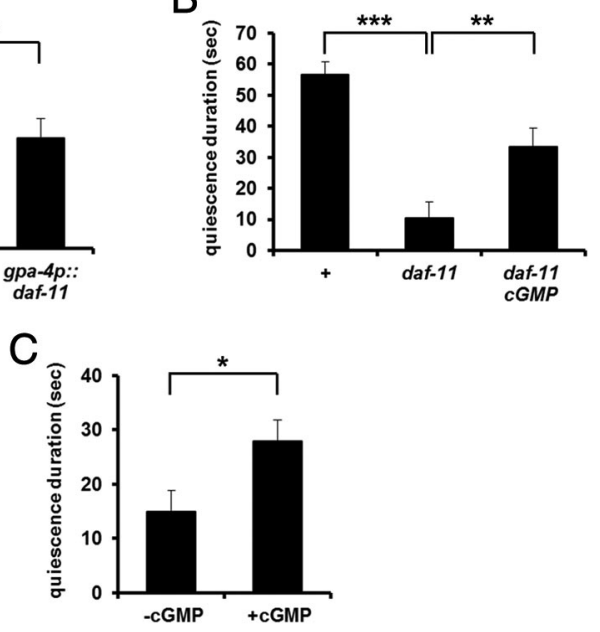

Figure 5. cGMP signaling in ASI promotes quiescence and inhibits dauer formation. $\boldsymbol{A}$, Expression of wild-type DAF-11 in ASI (daf-11 ASI) rescued the satiety quiescence defect of a daf-11 mutant. $n=10$ for each group per experiment, repeated three times. ${ }^{* *} p<0.01$ by Student's $t$ test. $\boldsymbol{B}$, Addition of 8-Br-cGMP (1 mm, daf-11 + CGMP) only during refeeding period rescued the satiety quiescence defect of a daf-11 mutant. $n=10$ for each group per experiment, repeated three times. ${ }^{* *} p<0.01,{ }^{* * *} p<0.001$, by ANOVA followed by Student's $t$ test. C, Addition of 8-Br-cGMP (1 mm) enhances satiety quiescence under nonfasted conditions. Individual worms were placed singly on HB101-seeded plates for $18 \mathrm{~h}$ from L4, and then the quiescence durations were measured. $n=10$ for each group, repeated three times. ${ }^{*} p<0.05$, by Student's $t$ test.

vation signal or that other starvation-signaling molecules released from RIM and RIC inhibit satiety quiescence.

\section{cGMP signaling acts both upstream and downstream of} DAF-7 TGF $\beta$

A cGMP signaling pathway consisting of a membrane bound form of guanyly cyclase (GCY) and cGMP-dependent protein kinase (PKG) regulate satiety quiescence (You et al., 2008). Recently, Valentino et al. (2011) showed that mice lacking the uroguanylin gene, which encodes a ligand for a membranebound guanylate cyclase that produces cGMP, became obese. They also found that the uroguanylin-GUCY2C (the receptor) system works as a canonical satiety signaling system: UROGUANYLIN is released from the gut to bind GUCY2C in hypothalamus. This novel cGMP signaling pathway for appetite control in mammals proves strong conservation in the control of food intake in animals.

The membrane guanylyl cyclase DAF-11 is expressed in at least five pairs of amphid neurons including ASI (Birnby et al., 2000). In addition, Murakami et al. (2001)showed that daf-7 expression in ASI is dependent on daf-11, suggesting that cGMP 
and TGF $\beta$ signals interact in ASI neurons. To test whether DAF-11 is the source of the cGMP signal in ASI neurons, we expressed it in ASI using a gpa-4 promoter (Jansen et al., 1999) and measured satiety quiescence. The transgene rescued the daf-11 defect (Fig. 5A). To confirm that the defect of daf-11 mutants in satiety quiescence is due to its inability to produce cGMP, we added a stable analog of cGMP, 8-bromo-cGMP (1 mM) (Birnby et al., 2000). Adding 8-bromo-cGMP rescued the daf-11 defect in satiety quiescence (Fig. 5B). 8-bromo-cGMP even enhanced quiescence in wild-type worms under conditions where wild-type worms show little quiescence (see Materials and Methods, Nonfasted test; Fig. 5C).

Together, our results suggest that cGMP signaling mediated by DAF-11 acts in ASI to induce satiety quiescence.

\section{PKG function for quiescence}

PKG (cGMP-dependent protein kinase) activity correlates with locomotive activity that usually represents explorative and food seeking behavior in many invertebrates (Osborne et al., 1997; Ben-Shahar et al., 2002; Fujiwara et al., 2002; Raizen et al., 2008). We found that mutations in the gene encoding cGMP-dependent protein kinase, egl-4, affect quiescence; loss of function mutants show no quiescence, whereas the gain of function mutation shows excessive quiescence (You et al., 2008). When we measured locomotive activity of egl-4 loss of function mutants during refeeding using our new method, we could confirm that egl-4 is required for satiety quiescence (Fig. 1D-F). Moreover, we found that compared with when they were fasted and refed high quality food egl-4 mutants further increased their locomotive activity when they were fed low quality food or when they were fed high quality food without fasting (Fig. 6A-C). These results indicate that even if egl-4 mutants are defective in satiety quiescence, they can still alter their locomotive activity according to food quality and feeding history. This suggests that there are factors contributing to nutritional status-dependent locomotive activity other than EGL-4.

Previously we reported that expression of wild-type EGL-4 in tax-4-expressing neurons rescues the quiescence defect of egl-4 mutant worms. Because ASI is one of these neurons, we tested whether expression of egl-4 only in ASI using a gpa-4 promoter (Jansen et al., 1999) can rescue the defect of egl-4 mutants in satiety quiescence. The transgenic lines that carry a wild-type copy of egl-4 expressed only in ASI partially rescue the egl-4 defect in satiety quiescence [Fig. 6H,I; egl-4; gpa-4p::egl-4(+)].

F

$\mathrm{H}$
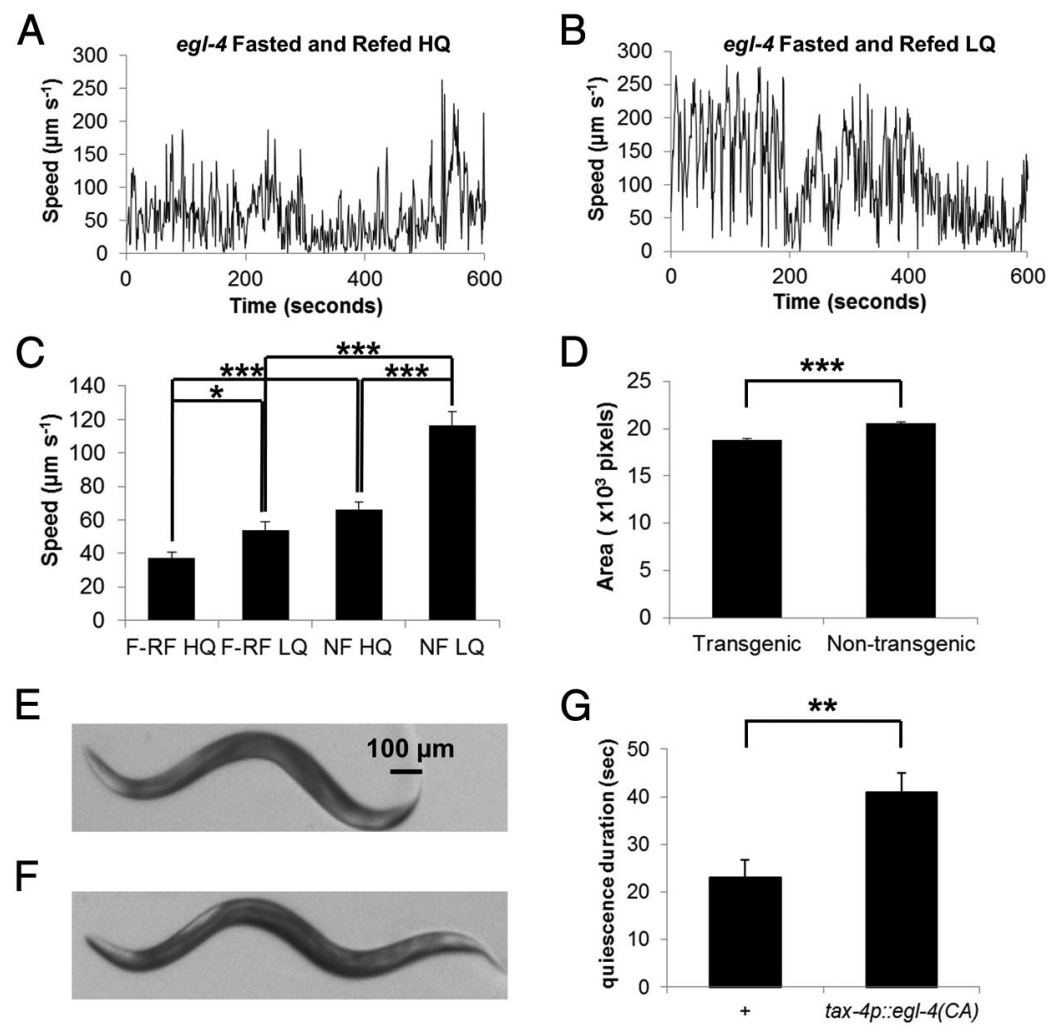

I
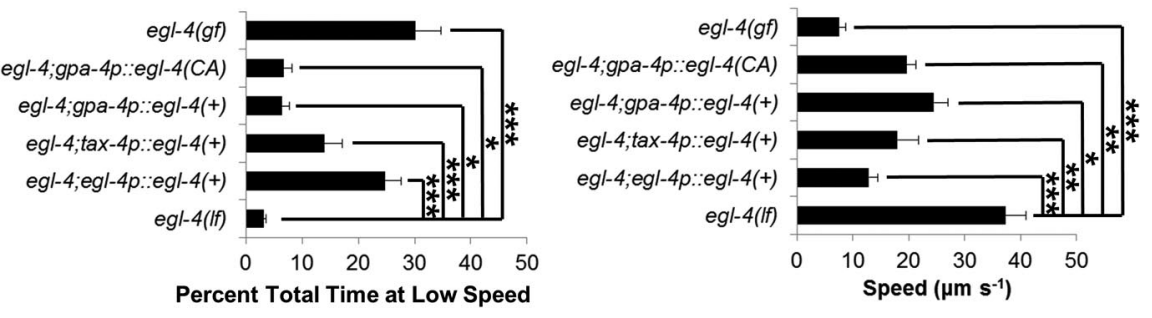

Figure 6. EGL-4 function for quiescence $A$, A representative speed plot of a fasted and refed egl-4 mutant worm on high quality food. $\boldsymbol{B}$, A representative speed plot of a fasted and refed egl-4 mutant worm on low quality food. $\boldsymbol{C}$, The mean speeds of egl-4 mutants under different conditions: F-RF HQ: fasted and refed high quality food, NF HQ: nonfasted and fed high quality food. F-RF LQ: fasted and refed low quality food. Fasted and refed high quality food of egl-4(If) was duplicated from Figure 1. At least 10 worms were tested for each group. ${ }^{*} p<0.05,{ }^{* *} p<0.01,{ }^{* * *} p<0.001$, Kruskal-Wallis ANOVA $(p<0.001)$ followed by Mann-Whitney $U$ test. $\boldsymbol{D}-\boldsymbol{F}$, Worms expressing EGL-4CA in TAX-4 neurons have smaller body size than their nontransgenic siblings, phenocopying egl-4(gf) worms. Quantification of body size (D) and representative pictures of a transgenic sibling $(\boldsymbol{E})$ and a nontransgenic sibling $(\boldsymbol{F})$. At least 30 worms were measured over two independent experiments. ${ }^{*} p<0.001$, by Student's $t$ test. $G$, Satiety quiescence duration is increased in worms expressing EGL-4CA in TAX-4 neurons (tax-4p::egl-4(CA)) compared with nontransgenic siblings after $12 \mathrm{~h}$ fasting and $3 \mathrm{~h}$ refeeding. $n=10$ for each group per experiment, repeated three times. ${ }^{* *} p<0.01$ by Student's $t$ test. $\boldsymbol{H}, \boldsymbol{I}$, Wild-type copy of egl-4 expressed under its own promoter or a tax- 4 promoter rescued the egl-4 defect in satiety quiescence with respect both to total time spent at low speed $\left(<1 \mu \mathrm{m} \mathrm{s}^{-1}, \mathrm{H}\right)$ and to mean speed $(I)$. However, wild-type egl-4 or egl-4CA expressed only in ASI neurons was not sufficient to fully rescue the egl-4 defect, especially in total time spent at low speed $(\boldsymbol{H})$, suggesting a partial rescue. ${ }^{*} p<0.05,{ }^{* *} p<0.01,{ }^{* * *} p<0.001$, Kruskal-Wallis ANOVA $(p<0.001)$ followed by Mann-Whitney $U$ test. egl-4(If) was duplicated from $C$ and Figure 1.

To further address EGL-4 function in ASI, we generated a constitutively active construct of EGL-4 and targeted its expression to ASI to examine whether constitutive activity of EGL-4 enhances satiety quiescence. We generated the constitutively active EGL- 4 by mutating serine 166 to aspartic acid. This S166D substitution (corresponding to S64D for mammalian PKG) mimics phosphorylation in the conserved autoinhibitory domain and is known to make the mammalian PKG constitutively active (Busch et al., 2002). To test whether this 
mutant indeed produced a gain-of-function effect, we expressed the mutated EGL-4 (EGL-4CA) in TAX-4-expressing neurons (AWB, AWC, ASE, ASI, ASJ, ASK, ASG, AFD, URX, and BAG) (Komatsu et al., 1996), which we previously showed to be the main sites of action of EGL-4 for satiety quiescence (You et al., 2008). The transgenic worms were smaller than nontransgenic siblings (Fig. $6 D-F$ ). This small body size phenocopies the previously isolated egl-4 gain of function mutant ad450 (Raizen et al., 2006). Furthermore, the transgenic worms showed enhanced quiescence compared with nontransgenic siblings under conditions where wild-type worms show little quiescence (see Materials and Methods, Nonfasted test), also mimicking ad450 (Fig. 6G). Expression of constitutively active EGL-4 in ASI rescues egl-4 partially; egl-4; ASI::egl-4CA worms showed a small increase in quiescence and decrease in roaming, but not to wild-type levels (Fig. 6H,I).

Together, these results suggest that EGL-4 can act in ASI to increase the time spent quiescent, probably at least in part by fully activating ASI, and thereby presumably increasing the release of DAF-7. However, it seems also to be required in neuron(s) other than ASI for normal levels of quiescence. In addition, egl-4 mutants still can exhibit different locomotive activity depending on their nutritional status, suggesting additional factors control nutritional status dependent locomotive activity.

\section{Discussion}

To survive in an unpredictable environment, animals have evolved to seek high quality food and eat as much as possible when food is available. Too much energy intake and too little expenditure causes the current pandemic of obesity that severely burdens society. Combining genetic and cell biology approaches, we showed that a satiety circuit consisting of ASI $\rightarrow$ RIM + RIC through the canonical TGF $\beta$-SMAD pathway promotes satiety quiescence in worms that mimics postprandial sleep in mammals. An unbiased mutant screen for a mutant defective in satiety quiescence yielded a new allele of $d a f-1$, a TGF $\beta$ receptor. Mutants of the TGF $\beta$-SMAD pathway are less quiescent and eat more. Ablation of ASI reduces satiety quiescence and increases food intake. Restoring the expression of $d a f-1$ in RIM and RIC rescues the daf-1 defect in satiety quiescence. In support of an ASI role in sensing nutritional status, calcium imaging shows that ASI is activated in the presence of nutrition. Together our result shows that ASI, known to play a critical role in calorie restrictiondependent longevity and in a developmental decision to survive a nutritionally harsh environment, also plays a key role in appetite control and satiety quiescence in worms. That the TGF $\beta$ pathway plays a significant role in connecting sensation of nutritional status to the animal's feeding behavior suggests a potential conservation between mammals and worms in the role of TGF $\beta$ in controlling appetite. In fact, overexpressing a TGF $\beta$ family member inhibits food intake in wild-type mice and causes weight loss by reducing food intake in leptin-deficient $o b / o b$ mice (Johnen et al., 2007). In addition, Sikder and Kodadek (2007) show that orexin, a neuropeptide that regulates appetite and sleep, upregulates expression of four sets of signaling genes including TGF $\beta$ / SMAD. These results implicate that neuronal TGF $\beta$ function in food intake and energy metabolism could be conserved.

Based on our results and the results of others, we propose a model (Fig. 7). Upon activation by nutrition, ASI releases DAF-7 TGF $\beta$. Binding of TGF $\beta$ to its receptor DAF-1 in RIM and/or RIC neurons inhibits their release of hunger signals. In the absence of hunger signals worms become satiated and quiescent (Fig. 7A). When food is not available, DAF-7 is not released and RIM and RIC are therefore activated to release hunger signals.
A

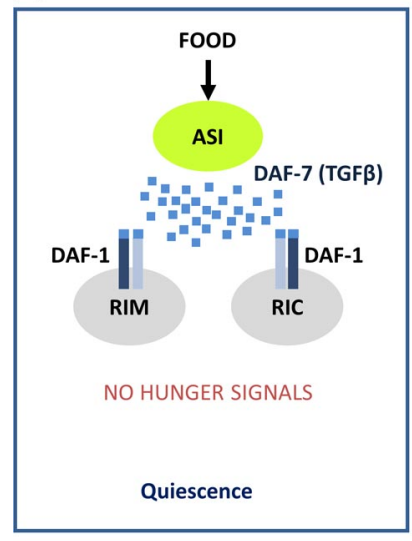

C
B

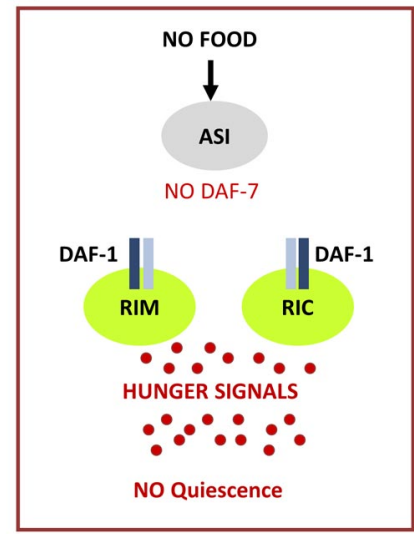

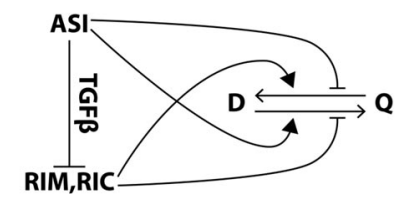

Figure 7. Model. $\boldsymbol{A}$, Upon activation by nutritional well being, ASI releases DAF-7 TGF $\beta$. Binding of TGF $\beta$ to its receptor DAF- 1 in RIM and RIC neurons inhibits RIM and RIC's role in releasing hunger signals. In the absence of hunger signals worms become satiated and quiescent. $\boldsymbol{B}$, When food is not available, DAF-7 is not released therefore RIM and RIC are activated to release hunger signals. C, A satiety circuit to control appetite in worms. D, Dwelling; $\mathbf{Q}$, quiescence.

This would wake worms up to explore for food (Fig. 7B). Therefore, "ASI $\rightarrow$ RIM + RIC" constitutes a satiety circuit to control appetite in worms (Fig. 7C).

Previous studies suggest that locomotive activity is tightly linked to an animal's nutritional state; hungry animals seek food and increase exploratory behavior, and satiated animals decrease exploratory behavior and take rest. Our development of an automated monitoring system combined with HMM analysis of quiescence (Gallagher et al., 2013) allowed us to discover that worms alternate periods of activity and inactivity during refeeding. In addition, using the analysis we found that ASI function is required for worms to show quiescence when worms are satiated; ASI specifically inhibits the switch from quiescence to dwelling and accelerates the switch from dwelling to quiescence thereby promoting quiescence. ASIablated worms and daf-7 mutants show an identical phenotype; they exit dwelling more frequently than wild-type therefore their total time spent in the quiescent state was reduced.

The identical phenotype of ASI-ablated worms and $d a f-7$ mutants suggests that the TGF $\beta$ pathway regulates food intake and satiety quiescence through ASI. DAF-7 and ASI provoke responses that are appropriate for a favorable nutritional state; they prevent dauer diapause, reduce fat accumulation, and enhance reproduction (Golden and Riddle, 1984b; Ren et al., 1996; Murakami et al., 2001; Li et al., 2003; Greer et al., 2008), all of which happen only in a good nutritional state. Quiescence is also achieved only in a good nutritional state. Therefore we can presume that activation of ASI and its release of DAF-7 could mean "good nutritional state" to worms. Interestingly we found that ASI is not activated by washed bacteria. This might suggest that ASI is not directly involved in detecting bacteria but involved in sensing nutritional state. How DAF-7 release and the activity of ASI correlate with actual transitions between states will require a long-term monitoring 
of neuronal activity of ASI. It will be an important question to understand how neurons integrate nutritional well being to control appetite and food intake.

It has been shown that a cGMP pathway regulates locomotive activity related to nutritional status (Osborne et al., 1997; Ben-Shahar et al., 2002; Fujiwara et al., 2002; Raizen et al., 2008; You et al., 2008). Our new automated monitoring system confirmed that egl-4 is absolutely required for satiety quiescence; we did not detect inactive locomotive periods in egl-4 mutants. Increased function of EGL-4 in ASI by a gain of function mutation enhances satiety quiescence, suggesting egl-4 function in ASI. However, egl-4 is required in other cells than ASI because expressing egl-4 only in ASI did not fully rescue the egl-4 mutant defect in satiety quiescence. This suggests that there are action sites other than ASI for EGL-4 to regulate satiety quiescence. Interestingly, an egl-4 mutant can still respond to the changes in nutritional status, such as difference in food quality. Because egl-4 mutants are completely incapable of showing satiety, this ability suggests that the increase of locomotion by low food quality can be caused by another signal likely coming from hunger. Our low quality food in fact made worms appear starved. It is interesting to speculate that the whole range of locomotive activity can be controlled by the integration of two types of signals: one to sense fullness and the other to sense hunger.

\section{References}

Alkema MJ, Hunter-Ensor M, Ringstad N, Horvitz HR (2005) Tyramine functions independently of octopamine in the Caenorhabditis elegans nervous system. Neuron 46:247-260. CrossRef Medline

Antin J, Gibbs J, Holt J, Young RC, Smith GP (1975) Cholecystokinin elicits the complete behavioral sequence of satiety in rats. J Comp Physiol Psychol 89:784-790. CrossRef Medline

Avery L (1993) The genetics of feeding in Caenorhabditis elegans. Genetics 133:897-917. Medline

Bargmann CI, Avery L (1995) Laser killing of cells in Caenorhabditis elegans. Methods Cell Biol 48:225-250. CrossRef Medline

Bargmann CI, Horvitz HR (1991) Control of larval development by chemosensory neurons in Caenorhabditis elegans. Science 251:1243-1246. CrossRef Medline

Ben Arous J, Laffont S, Chatenay D (2009) Molecular and sensory basis of a food related two-state behavior in C. elegans. PLoS One 4:e7584. CrossRef Medline

Ben-Shahar Y, Robichon A, Sokolowski MB, Robinson GE (2002) Influence of gene action across different time scales on behavior. Science 296:741744. CrossRef Medline

Beverly M, Anbil S, Sengupta P (2011) Degeneracy and neuromodulation among thermosensory neurons contribute to robust thermosensory behaviors in Caenorhabditis elegans. J Neurosci 31:11718-11727. CrossRef Medline

Birnby DA, Link EM, Vowels JJ, Tian H, Colacurcio PL, Thomas JH (2000) A transmembrane guanylyl cyclase (DAF-11) and Hsp90 (DAF-21) regulate a common set of chemosensory behaviors in caenorhabditis elegans. Genetics 155:85-104. Medline

Busch JL, Bessay EP, Francis SH, Corbin JD (2002) A conserved serine juxtaposed to the pseudosubstrate site of type I cGMP-dependent protein kinase contributes strongly to autoinhibition and lower cGMP affinity. J Biol Chem 277:34048-34054. CrossRef Medline

Chelur DS, Chalfie M (2007) Targeted cell killing by reconstituted caspases. Proc Natl Acad Sci U S A 104:2283-2288. CrossRef Medline

Chronis N, Zimmer M, Bargmann CI (2007) Microfluidics for in vivo imaging of neuronal and behavioral activity in Caenorhabditis elegans. Nat Methods 4:727-731. CrossRef Medline

Crocker A, Shahidullah M, Levitan IB, Sehgal A (2010) Identification of a neural circuit that underlies the effects of octopamine on sleep-wake behavior. Neuron 65:670-681. CrossRef Medline

Elmquist JK, Elias CF, Saper CB (1999) From lesions to leptin: hypothalamic control of food intake and body weight. Neuron 22:221-232. CrossRef Medline
Fujiwara M, Sengupta P, McIntire SL (2002) Regulation of body size and behavioral state of C. elegans by sensory perception and the EGL-4 cGMPdependent protein kinase. Neuron 36:1091-1102. CrossRef Medline

Gallagher T, Bjorness T, Greene R, You Y, Avery L (2013) The geometry of locomotive behavioral states in C. elegans. PLoS One 8(3):e59865. CrossRef Medline

Golden JW, Riddle DL (1984a) The Caenorhabditis elegans dauer larva: developmental effects of pheromone, food, and temperature. Dev Biol 102: 368-378. CrossRef Medline

Golden JW, Riddle DL (1984b) A pheromone-induced developmental switch in Caenorhabditis elegans: temperature-sensitive mutants reveal a wild-type temperature-dependent process. Proc Natl Acad Sci U S A 81: 819-823. CrossRef Medline

Greer ER, Pérez CL, Van Gilst MR, Lee BH, Ashrafi K (2008) Neural and molecular dissection of a $C$. elegans sensory circuit that regulates fat and feeding. Cell Metab 8:118-131. CrossRef Medline

Gunther CV, Georgi LL, Riddle DL (2000) A Caenorhabditis elegans type I TGF beta receptor can function in the absence of type II kinase to promote larval development. Development 127:3337-3347. Medline

Hetherington AW, Ranson SW (1940) Hypothalamic lesions and adiposity in the rat. Anat Rec 78:149-172. CrossRef

Hu PJ (2007) Dauer. In: WormBook: the online review of C elegans biology. http://www.wormbook.org/.

Jansen G, Thijssen KL, Werner P, van der Horst M, Hazendonk E, Plasterk RH (1999) The complete family of genes encoding G-proteins of Caenorhabditis elegans. Nat Genet 21:414-419. CrossRef Medline

Johnen H, et al (2007) Tumor-induced anorexia and weight loss are mediated by the TGF-beta superfamily cytokine MIC-1. Nat Med 13:13331340. CrossRef Medline

Komatsu H, Mori I, Rhee JS, Akaike N, Ohshima Y (1996) Mutations in a cyclic nucleotide-gated channel lead to abnormal thermosensation and chemosensation in C. elegans. Neuron 17:707-718. CrossRef Medline

Li S, Dent JA, Roy R (2003) Regulation of intermuscular electrical coupling by the Caenorhabditis elegans innexin inx-6. Mol Biol Cell 14:2630-2644. CrossRef Medline

Murakami M, Koga M, Ohshima Y (2001) DAF-7/TGF-beta expression required for the normal larval development in C. elegans is controlled by a presumed guanylyl cyclase DAF-11. Mech Dev 109:27-35. CrossRef Medline

Osborne KA, Robichon A, Burgess E, Butland S, Shaw RA, Coulthard A, Pereira HS, Greenspan RJ, Sokolowski MB (1997) Natural behavior polymorphism due to a cGMP-dependent protein kinase of Drosophila. Science 277:834-836. CrossRef Medline

Patterson GI, Padgett RW (2000) TGF beta-related pathways. Roles in Caenorhabditis elegans development. Trends Genet 16:27-33. CrossRef Medline

Raizen DM, Cullison KM, Pack AI, Sundaram MV (2006) A novel gain-offunction mutant of the cyclic GMP-dependent protein kinase egl-4 affects multiple physiological processes in Caenorhabditis elegans. Genetics 173: 177-187. CrossRef Medline

Raizen DM, Zimmerman JE, Maycock MH, Ta UD, You YJ, Sundaram MV, Pack AI (2008) Lethargus is a Caenorhabditis elegans sleep-like state. Nature 451:569-572. CrossRef Medline

Raizen D, Song BM, Trojanowski N, You YJ (2012) Methods for measuring pharyngeal behaviors. WormBook 18:1-13. CrossRef Medline

Ren P, Lim CS, Johnsen R, Albert PS, Pilgrim D, Riddle DL (1996) Control of C. elegans larval development by neuronal expression of a TGF-beta homolog. Science 274:1389-1391. CrossRef Medline

Shtonda BB, Avery L (2006) Dietary choice behavior in Caenorhabditis elegans. J Exp Biol 209:89-102. CrossRef Medline

Sikder D, Kodadek T (2007) The neurohormone orexin stimulates hypoxiainducible factor-1 activity. Genes Dev 21:2995-3005. CrossRef Medline

Sulston JE, Hodgkin JG (1988) Methods. In: The nematode Caenorhabditis elegans (Wood, W. B., ed), pp 587-606. New York: Cold Spring Harbor.

Suo S, Kimura Y, Van Tol HH (2006) Starvation induces cAMP response elementbinding protein-dependent gene expression through octopamine-Gq signaling in Caenorhabditis elegans. J Neurosci 26:10082-10090. CrossRef Medline

Suzuki H, Kerr R, Bianchi L, Frøkjaer-Jensen C, Slone D, Xue J, Gerstbrein B, Driscoll M, Schafer WR (2003) In vivo imaging of C. elegans mechanosensory neurons demonstrates a specific role for the MEC-4 channel in the process of gentle touch sensation. Neuron 39:1005-1017. CrossRef Medline 
Valentino MA, Lin JE, Snook AE, Li P, Kim GW, Marszalowicz G, Magee MS, Hyslop T, Schulz S, Waldman SA (2011) A uroguanylin-GUCY2C endocrine axis regulates feeding in mice. J Clin Invest 121:3578-3588. CrossRef Medline
You YJ, Kim J, Raizen DM, Avery L (2008) Insulin, cGMP, and TGF-beta signals regulate food intake and quiescence in C. elegans: a model for satiety. Cell Metab 7:249-257. CrossRef Medline 